\title{
Optimizing SHIELD-HIT for carbon ion treatment
}

\author{
David C Hansen ${ }^{1,2}$, Armin Lühr ${ }^{1,2}$, Nikolai Sobolevsky ${ }^{3}$ and \\ Niels Bassler ${ }^{1,2}$ \\ ${ }^{1}$ Department of Experimental Clinical Oncology, Aarhus University Hospital, \\ Nørrebrogade 44 Bldg. 5, DK-8000 Aarhus C, Denmark \\ 2 Department of Physics and Astronomy, Aarhus University, Ny Munkegade 120, \\ DK-8000 Aarhus C, Denmark \\ ${ }^{3}$ Department of Neutron Research, Institute for Nuclear Research of the Russian \\ Academy of Sciences, 7a, 60th October Anniversary prospect, Moscow 117312, Russia \\ E-mail: dch@ki.au.dk
}

\begin{abstract}
The SHIELD-HIT Monte Carlo Transport code has been widely used in particle therapy, but has previously shown some discrepancies, when compared with experimental data. In this work, the inelastic nuclear cross sections of SHIELD-HIT are calibrated to experimental data for carbon ions. In addition, the models for nuclear fragmentation were adjusted to experiments, for the partial charge changing cross section of carbon ions in water. Comparison with fragmentation yield experiments for carbon and neon primaries were made for validation. For carbon primaries, excellent agreement between simulation and experiment was observed, with only minor discrepancies. For neon primaries, the agreement was also good, but larger discrepancies were observed, which require further investigation. In conclusion, the current version SHIELD-HIT10A is well suited for simulating problems arising in particle therapy for clinical ion beams.
\end{abstract}

PACS numbers: 87.10.Rt, 87.55.K-, 87.55.Qr, 24.10.Lx

Keywords: Carbon ion therapy, Monte Carlo Transport, Nuclear fragmentation, SHIELD-HIT

Submitted to: Phys. Med. Biol.

\section{Introduction}

The application of Monte Carlo (MC) transport codes in particle therapy research covers a broad range of topics. Common for all topics is that they require detailed knowledge about the particle beams used clinically. Such knowledge can be obtained by solving the Boltzmann transport equation. However, doing this analytically is not possible for anything but the simplest geometries and beams. Therefore, the only way to obtain knowledge about the particle-energy spectrum in a given point is either by 
experiment or by solving the transport equation by the Monte Carlo method. Most ion treatment planning systems work with pencil beam like algorithms where pre-calculated pencil beam dose kernels are applied on a voxel-based structure. Such dose kernels can be calculated with MC codes where the full beam line is modelled. However, pencil beam algorithms with pre-calculated dose kernels are known to be inaccurate at media boundaries with strong density gradients, such as bone/tissue interfaces, lung or even if metal artefacts are in the treatment field (Schaffner et al. 1999). As computer power becomes cheaper, a full MC simulation can be realized which will give a more realistic treatment plan. Some developers of commercial treatment planning software offer this as an option for photon/electron treatment planning. Moreover, MC codes allow detailed theoretical studies of new types of particle beams for radiotherapy. This is in particular important for exotic particles where high current beams may not be available for experiments and clinical facilities are far from being realized, as in the case of antiproton radiotherapy (Bassler et al. 2010b, Bassler et al. 2008b, Bassler et al. 2008a, Bassler \& Holzscheiter 2009).

Apart from dose planning, dose assessment is also relying on $\mathrm{MC}$ calculations. In a recent study, the stopping power ratios used for ionization chamber dosimetry were investigated. Using the SHIELD-HIT MC code it was found that nuclear fragmentation only had a minor influence for the mixed particle field created by carbon ion beams (Lühr et al. 2011a), when following the formalism of IAEA TRS-398 protocol (IAEA TRS398 2000).

For calculating the radiobiological properties of the applied heavy ion beam, the Local Effect Model (LEM) (Krämer et al. 2000, Krämer \& Scholz 2000) can be used during the optimization of the treatment plan. The LEM, however, needs the particleenergy spectrum as an input parameter. These purely physical input parameters can either be derived experimentally or calculated by MC codes. Furthermore, dose delivery verification using either PET or prompt gamma rays (Parodi et al. 2007) relies on MC codes that can accurately simulate the distribution of radioactive nuclei after irradiation of the patient.

Finally, the generation of secondary neutrons which increases the risk of developing a secondary radiation-induced cancer has been subject of much discussion. Here MC codes are used to model the neutron fluence emerging from beam nozzles and the patient itself (Hall 2006, Xu et al. 2008), and again for research purposes of more exotic beam environments (Bassler et al. 2010a)

For ion beams Monte Carlo transport codes such as Geant4 (Agostinelli et al. 2003, Allison et al. 2006), FLUKA (Fassò et al. 2005, Battistoni et al. 2007), PHITS (Niita et al. 2006, Iwase et al. 2002), MCNPX (Pelowitz 2005) and SHIELD-HIT (Gudowska et al. 2004, Geithner et al. 2006) are frequently used. These codes all rely on sophisticated models for nuclear interactions, which have to balance speed and accuracy, while remaining as general in terms of energy and particle type as possible. SHIELDHIT is a Monte Carlo code specialized for ion therapy. However, previous studies have suggested that there were some inaccuracies in the nuclear models implemented 
in SHIELD-HIT when comparing to experimental fragmentation yields (Gudowska et al. 2004). Since then several updates were made to SHIELD-HIT, and the more recent changes will be treated in the next section. The purpose of this paper is thus to give a presentation of the recent improvements of the nuclear models in SHIELD-HIT10A and a new benchmark of the underlying nuclear models against available experimental data.

The SHIELD-HIT Monte Carlo transport code (Gudowska et al. 2004) is derived from the SHIELD code (Dementyev \& Sobolevsky 1999), originally developed at the Joint Institute for Nuclear Research (JINR), in Dubna, Russia. Later, development was moved to the Institute for Nuclear Research (INR) at the Russian Academy of Sciences in Moscow. The SHIELD code includes transport of arbitrary nuclei, nucleons, antinucleons, pions and kaons up to $1 \mathrm{TeV} / u$ and down to $1 \mathrm{MeV} / u$. In SHIELD-HIT, the same particles can be transported, but here the upper and lower limit ranges from $2 \mathrm{GeV} / u$ to $25 \mathrm{keV} / u$. Previous to this work, the latest version of SHIELD-HIT was 08. In SHIELD-HIT10A several parameters regarding the nuclear reactions were changed along with several updates to the user interface and performance, which were partially discussed in (Hansen et al. 2012).

SHIELD-HIT has earlier been used for various tasks within particle therapy, calculation of stopping power ratios (Henkner et al. 2009, Lühr et al. 2011a, Lühr et al. 2012), fluence correction factors (Lühr et al. 2011b), and even antiproton beams (Bassler et al. 2008b).

In this article, we focus on the changes to the nuclear models used in SHIELD-HIT. The next section will give a more detailed description of the parameters of the nuclear interaction models as well as how SHIELD-HIT works in general.

\section{The SHIELD-HIT Monte Carlo code}

Before starting the Monte Carlo transport in SHIELD-HIT the stopping power, cross sections, ranges and optical depths are calculated for all the materials in the simulation at various energy intervals. These tables are then used for interpolation of the values during the transport phase, using linear or quadratic interpolation. The energy grids used in the tables were refitted from SHIELD to SHIELD-HIT to yield the accuracy required in medical applications.

\subsection{Electronic interactions}

Stopping powers in SHIELD-HIT are calculated using the Bethe-Bloch formula (Fano 1963), with effective charge correction (Hubert et al. 1989) but without the shellcorrection terms. For energies below the stopping power maximum, the LindhardScharff equation (Lindhard \& Scharff 1961) is used. The exact energy where SHIELDHIT goes from Bethe-Bloch to Lindhard-Scharff is determined by the requirement that the stopping power must remain a smooth function. Alternatively, the stopping power can be interpolated from external tables, following the ICRU format, on an individual 
material basis. The software library libdEdx (Toftegaard et al. 2010, Lühr et al. 2012) allows direct use of the PSTAR, ASTAR (Berger et al. 2005, Paul \& Schinner 2001, Paul \& Schinner 2002), and ICRU stopping power tables in SHIELD-HIT, as it directly exports in the required text format. In this work, the stopping power tables published in the ICRU report 49 and 73 (with erratum) (ICRU Report 49 1993, ICRU Report 73 2005) are used for water and air, and the Bethe-Bloch equation for any other material. SHIELD-HIT includes multiple Coulomb scattering through two dimensional Fermi (Gaussian) or Molière distributions (Bethe 1953) and fluctuations of ionization energy losses in the form of Gaussian or Vavilov energy straggling. In this work, Molière and Vavilov distributions were used for all calculations where applicable.

\subsection{Nuclear interactions}

It is common in Monte Carlo transport codes to handle nuclear interactions in two separate steps. First, the probability that a nuclear event happens is sampled, based on the total inelastic nuclear cross section. Then, if an event happens, a separate model for nuclear fragmentation is used to sample the outcome and get the fragmentation yields.

2.2.1. Total inelastic cross sections In SHIELD-HIT up to version 08, the total inelastic cross sections for nuclear interactions are taken from (Barashenkov 1993). The nucleon-ion collision cross-sections are interpolated from tables based on experiments and theoretical calculations. These tables exist for 18 reference nuclei and non-tabulated isotopes are then interpolated linearly weighted with the geometrical nucleus cross section. For an element with nucleon number $A$ this is assumed to be a factor $A^{2 / 3}$. For ion-ion collisions, the cross sections are given by a strong absorption model type equation:

$$
\begin{aligned}
\sigma_{R}(E) & =\pi r_{0}^{2}\left(1-\frac{B(E)}{E_{\mathrm{cm}}}\right) \\
& \left(A_{P}^{1 / 3}+A_{T}^{1 / 3}+1.85 \frac{A_{P}^{1 / 3} A_{T}^{1 / 3}}{A_{P}^{1 / 3}+A_{T}^{1 / 3}}+\lambda(A, E)-\delta_{B}(E)\right)^{2}
\end{aligned}
$$

where $r_{0}$ is the nucleon radius set to $=1.3 \mathrm{fm}, E$ is the kinetic energy in the laboratory frame, $E_{\mathrm{cm}}$ is kinetic energy in the centre of mass frame, $\lambda(A, E)$ is the de Broglie wavelength of the projectile in the centre of mass system and $A_{P}$ and $A_{T}$ are the projectile and target nucleon numbers, respectively. $B(E)$ is the Coulomb barrier given by

$$
B(E)=\frac{1}{4 \pi \epsilon_{0}} \frac{Z_{P} Z_{T}}{r_{0}\left(A_{P}^{1 / 3}+A_{T}^{1 / 3}\right)+\lambda(A, E)}
$$

where $Z_{P}$ and $Z_{T}$ are the atomic numbers of the projectile and target, respectively, and

$$
\delta_{B}(E)= \begin{cases}C_{1}+C_{2} \log \left(\frac{E}{A_{T}}\right) & \text { for } \frac{E}{A_{T}}<E_{1}, \\ C_{3}+C_{4} \log \left(\frac{E}{A_{T}}\right) & \text { for } E_{1} \leq \frac{E}{A_{T}}<E_{2}, \\ C_{5} & \text { for } E_{2} \leq \frac{E}{A_{T}}\end{cases}
$$


where $C_{1}, C_{2}, C_{3}, C_{4}, C_{5}, E_{1}$, and $E_{2}$ are model parameters. For $E / A_{T} \leq 10 \mathrm{MeV} / u$, the cross section for $E / A_{T}=10 \mathrm{MeV} / u$ is used, but scaled with the correct Coulomb barrier given by equation 2 .

2.2.2. Nuclear fragmentation Nuclear fragmentation in SHIELD-HIT is handled by the Many Stage Dynamical Model (MSDM) (Botvina et al. 1997). This model consists of 3 steps: fast-cascade, pre-equilibrium and de-excitation/equilibrium. In the initial fast-cascade, it is assumed that all long range interactions can be neglected and the time evolution of the system is dominated by short range interactions (i.e. collisions). For energies below $1 \mathrm{GeV} / u$ it is done using the Dubna-cascade model (Toneev \& Gudima 1983) and for higher energies it is handled by the Quark-gluon string model (Amelin et al. 1990).

The second step is called the pre-equilibrium step, which is an exciton model. Here the excited nucleus is treated as a particle-hole system and solved using the Boltzmann Master Equation (Gudima et al. 1983) (BME). This takes the long range interactions into account but neglects the collision geometry.

The last step is called the de-excitiation or equilibrium step, where one of 3 different models is used. For fragments with nucleon number $A \leq 16$, the Fermi-breakup model (Fermi 1950, Botvina et al. 1987) is applied. For heavier fragments $A>16$ which are excited to less than $2 \mathrm{MeV} / u$ an evaporation model (Botvina et al. 1987) is used and otherwise the statistical model of multifragmentation (Botvina et al. 1987, Bondorf et al. 1995). For ion therapy the energies currently used are mainly below $400 \mathrm{MeV} / u$. At these energies only a negligible small fraction of the simulated secondary particles reach an energy above $1 \mathrm{GeV} / u$, and thus only the influence of the Dubna cascade was considered in this work. Since carbon ions and possibly oxygen ions are the heaviest projectiles used for treatment, and the human body primarily consists of relatively light elements, the Fermi-breakup model is dominating the de-excitation step. For heavier

ions, which have been proposed for treatment, such as oxygen and neon, the evaporation model becomes relevant.

\section{Changes in the nuclear models of SHIELD-HIT}

\subsection{Corrections of the total cross sections}

The tabulated proton-ion cross sections implemented in SHIELD-HIT08 agree well with experiments (most within $2 \sigma$ ), and can be considered sufficiently accurate. Thus, these cross-sections were not changed in SHIELD-HIT10A.

In contrast, the agreement for the ion-ion cross sections based on the analytical parametrization was less convincing, and adjustments were needed. The total inelastic cross sections were changed by simply calibrating the parameters of the Barashenkov cross section formula (equation 1) to experimental data for carbon ions (Takechi et al. 2009, Fang et al. 2000, Kox et al. 1987, Sihver et al. 1993, Zhang et al. 2002). This 


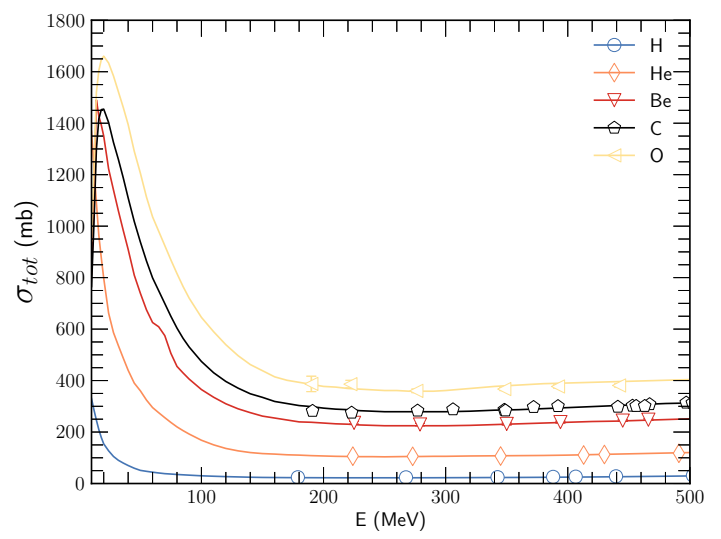

Figure 1: Total nuclear cross sections in SHIELD-HIT for protons on various targets, compared with experimental data (Schwaller et al. 1979).

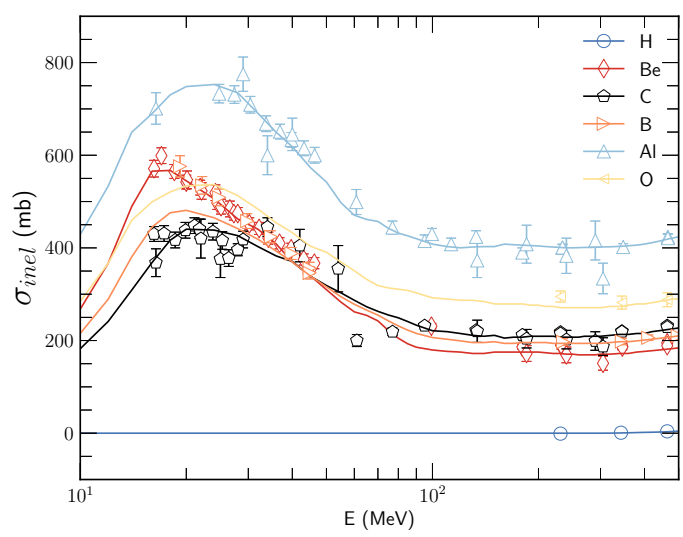

Figure 2: Inelastic nuclear cross sections in SHIELD-HIT for protons on various targets, compared with experimental data (Sihver et al. 1993).

was done using a coarse brute-force optimization and then finding the local minimum with the Simplex-Downhill algorithm (Powell 1973). Carbon was used for the calibration for several reasons: First of all due to its role as the dominant ion in heavy ion therapy. Second, carbon is relatively easy to use in both as a primary beam and as a target in experiments, so relatively many data sets are available. The Tripathi (Tripathi et al. 1996, Tripathi et al. 1997, Tripathi et al. 1999) cross sections used in Geant4 were also considered for comparison, as the experimental data used in the calibration cannot also serve as validation.

3.1.1. Proton-nucleus cross sections As can be seen on figures 1 and 2 , the total and inelastic cross sections for protons fit well with available experimental data. A notable exception is the inelastic cross section for boron, where some discrepancies can be observed below $40 \mathrm{MeV} / u$. This is most likely because the boron cross sections are interpolated from the tables of carbon and beryllium which is not sufficiently accurate at low energies. Some disagreement can also be observed for the inelastic proton-carbon cross section, but here the experimental data is less conclusive.

3.1.2. Ion-nucleus cross sections When comparing SHIELD-HIT08 with the experimental data for carbon on various targets (figures 3 and 4), a marked overestimation of the cross section is observed in the region of about $100 \mathrm{MeV} / u$ to $500 \mathrm{MeV} / u$ for $\mathrm{C}$, Ne and $\mathrm{Al}$ targets and to a minor degree for Be targets as well. A similar, but less pronounced overestimation is seen in the Tripathi (Geant4) data set. Optimizing the constants in the Barashenkov formula to the available experimental data yielded the values in table 1 . It was not possible to make the data for beryllium fit using the same constants as for the heavier materials, so a separate set of constants 


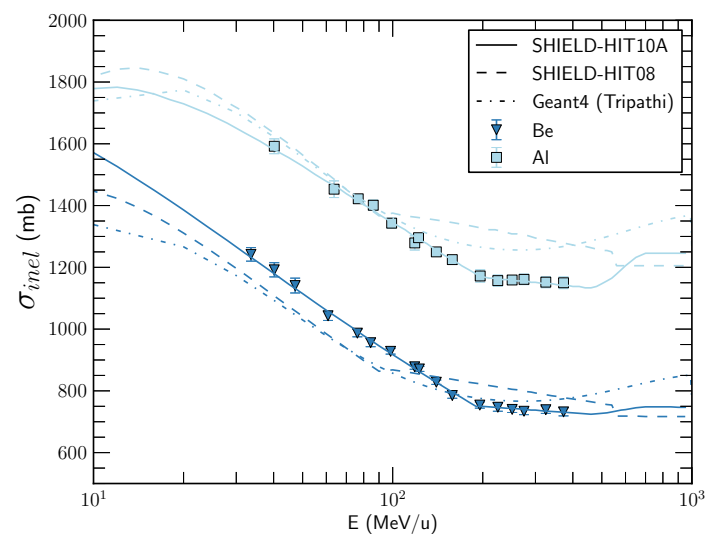

Figure 3: Inelastic nuclear cross sections from Geant4 (Tripathi), SHIELD-HIT08 and SHIELD-HIT10A for ${ }^{12} \mathrm{C}$ on beryllium and aluminium targets compared with experimental data (Takechi et al. 2009, Kox et al. 1984, Kox et al. 1985).

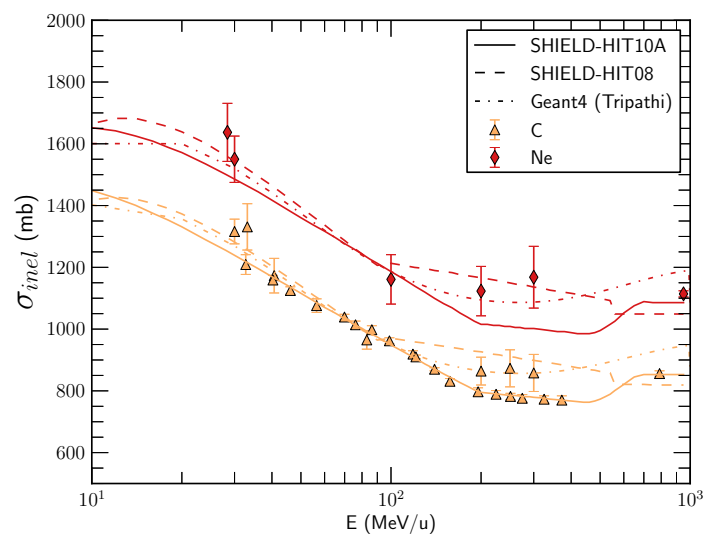

Figure 4: Inelastic nuclear cross sections from Geant4 (Tripathi), SHIELD-HIT08 and SHIELD-HIT10A for ${ }^{12} \mathrm{C}$ on carbon and neon targets, compared with experimental data (Takechi et al. 2009, Fang et al. 2000, Kox et al. 1984, Kox et al. 1985, Kox et al. 1987, Sihver et al. 1993, Zhang et al. 2002).

\begin{tabular}{|c|c|c|c|c|c|c|c|c|}
\hline \multirow{2}{*}{ SHIELD-HIT08 } & & $C_{1}$ & $C_{2}$ & $E_{1}$ & $C_{3}$ & $C_{4}$ & $E_{2}$ & $C_{5}$ \\
\cline { 2 - 9 } & $Z>2$ & -2.05 & 1.9 & $100 \mathrm{MeV} / u$ & 0.8 & 0.426 & $560 \mathrm{MeV} / u$ & 2.07 \\
\hline \multirow{2}{*}{ SHIELD-HIT10 } & $Z>4$ & -1.112 & 1.427 & $195 \mathrm{MeV} / u$ & 1.624 & 0.2336 & $560 \mathrm{MeV} / u$ & 1.98 \\
\cline { 2 - 9 } & $2<Z \leq 4$ & -1.768 & 1.652 & $195 \mathrm{MeV} / u$ & 1.585 & 0.178 & $560 \mathrm{MeV} / u$ & 1.98 \\
\hline
\end{tabular}

Table 1: Parameters used in the Barashenkov cross section model (equation 1) for inelastic nucleus-nucleus collisions in SHIELD-HIT08 and SHIELD-HIT10A.

were used for beryllium and lithium. This way, excellent agreement between SHIELDHIT10A and the experimental cross sections could be achieved. The parametrization of $\sigma_{R}(E)$ in equation 1 with the parameter in table 1 produces an unphysical jump in the cross section at $560 \mathrm{MeV} / u$, and the cross sections from $450 \mathrm{MeV} / u$ to $700 \mathrm{MeV} / u$ were computed using spline interpolation to ensure a smooth transition. It was not possible to verify the applied smoothing as no data was available in this region and the Tripathi cross section shows no similar transition in this region. A possible way to compensate for the lack of experimental data would be to compare the data with non-empirical theoretical models such as optical limit Glauber calculations (Takechi et al. 2009) being, however, beyond the scope of the current work. 


\subsection{Corrections to the models for nuclear fragmentation}

The steps in the MSDM model for nuclear fragmentation are highly interdependent, and therefore they are difficult to validate against experimental data separately. Conversely, it is difficult to isolate any discrepancies to a single step. The Fermi-breakup model is the dominating model in the final step and therefore deserves closer attention. This model requires calibration of two parameters known as the relative free volume $\left(\frac{V_{f r}}{V_{0}}\right)$ and the relative free Coulomb volume $\left(\frac{V_{f r}^{c}}{V_{0}}\right)$. The order of magnitude of these parameters is physically motivated (Fermi 1950) and they are usually set to 1.0. Changing the free volume to larger values would result in more, but lighter fragments being produced. A similar behaviour is seen for the Coulomb volume, but it is more dependent on the energy and excitation of the fragment. The Fermi-breakup model was also recently validated in Geant4 (Pshenichnov et al. 2010).

In this work, the model was calibrated to experimental partial charge-changing cross sections from a ${ }^{12} \mathrm{C}$-beam on water (Toshito et al. 2007, Golovchenko et al. 2002). The partial charge-changing cross section is the cross section for a process, in which a fragment is produced with a specific charge. A program was created to simulate these two experiments for a given projectile on a pure element with the naturally occurring isotope content. This was done by simulating 100000 collisions for every energy and then multiplying the obtained partial fragmentation yields with the corresponding inelastic cross section. For composite targets such as water, the results for the basic elements were combined by simply adding the cross sections for the elements contained in the molecule. In order to compare with what could be detected in experiments, fragments with an energy below $25 \mathrm{MeV} / u$ were filtered out. For carbon this corresponds to a residual range of about $2 \mathrm{~mm}$ in water or $2 \mathrm{~m}$ in air. Performing the calibration with computer optimization was impractical due to the time required to calculate the partial charge-changing cross sections. Instead, the optimization of $\frac{V_{f r}^{c}}{V_{0}}$ and $\frac{V_{f r}}{V_{0}}$ was done manually, against partial charge-changing cross sections of ${ }^{12} \mathrm{C}$ on water. Water was chosen for two reasons. First, it is the standard reference material for radiotherapy and second, most of the experimental data was available for this material.

3.2.1. Comparison with partial charge-changing cross section experiments The Fermibreakup volumes best fitting the experimental data and therefore used in SHIELDHIT10A were found to be $\frac{V_{f r}^{c}}{V_{0}}=0.65$ and $\frac{V_{f r}}{V_{0}}=18.0$. As previously mentioned, the nuclear models are highly interdependent. Thus, these values are only valid for the Fermi-breakup in combination with the cascade and pre-equilibrium models used in SHIELD-HIT and may compensate for inconsistencies in these two models. In combination with other models a different set of breakup volumes might be appropriate. Comparison of the partial charge-changing cross sections of ${ }^{12} \mathrm{C}$ between SHIELDHIT10A SHIELD-HIT08 and experimental data can be seen in figures 5 and 6 . SHIELDHIT08 is greatly overestimating the $\Delta Z=2$ and $\Delta Z=3$ channels for both PMMA and water when compared with experimental data, but the $\Delta Z=1$ channel is in relatively 


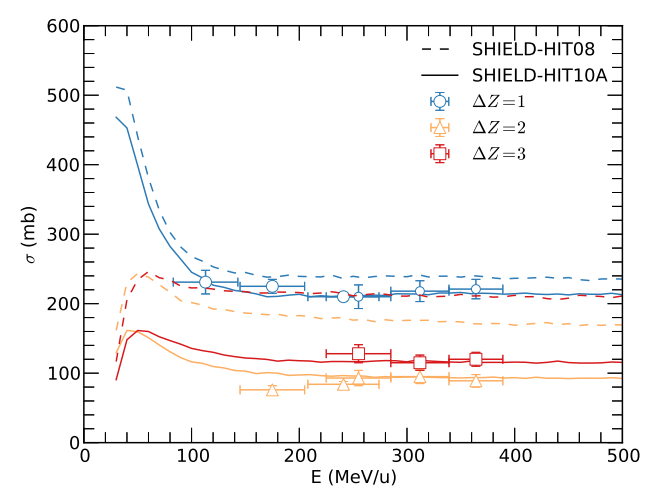

Figure 5: Partial charge-changing cross section of ${ }^{12} \mathrm{C}$ on water for SHIELD-HIT08 and SHIELD-HIT10A compared with the experimental data (Toshito et al. 2007, Golovchenko et al. 2002) used for adjusting SHIELD-HIT10A.

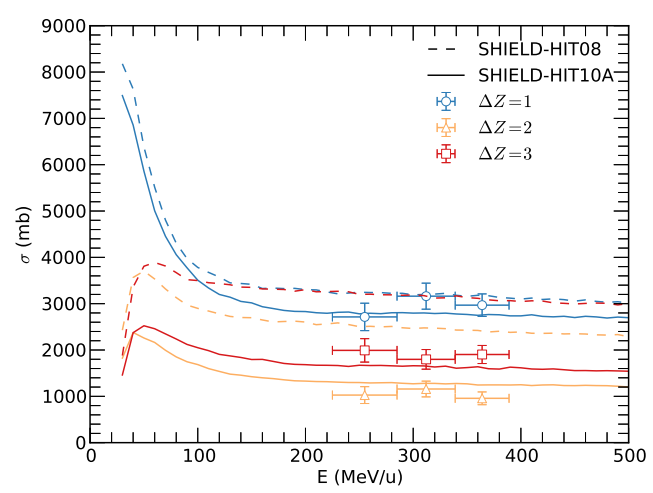

Figure 6: Partial charge-changing cross section of ${ }^{12} \mathrm{C}$ on PMMA for SHIELD-HIT08 and SHIELD-HIT10A compared with experimental data (Toshito et al. 2007).

good agreement. For SHIELD-HIT10A good agreement could be achieved with the data to which it was optimized (figure 5). The lower cross sections seen experimentally below $250 \mathrm{MeV} / u$ for $\Delta Z=2$ are not reproduced by the fragmentation model, however, it is possible that they are artefacts coming from a systematic difference between the different experiments included. In order to validate the adjustments of the charge-changing cross sections, SHIELD-HIT10A was compared with experimental data for PMMA (figure 6). It can be seen that SHIELD-HIT10A is in good agreement and within $2 \sigma$ of the data everywhere.

\section{Comparison with fragmentation experiments}

To validate the changes made in SHIELD-HIT10A an extensive comparison with experimental data is required. A detailed experimental investigation of nuclear fragmentation of $400 \mathrm{MeV} / u$ carbon ions has been made at GSI (Haettner 2006, Haettner et al. 2006). This data is particularly well suited for benchmarking SHIELD-HIT as it focuses on the fragmentation occurring in carbon ion therapy.

In the experiment, a $400 \mathrm{MeV} / u$ carbon ion beam was shot at a water phantom of variable length. The energies and time of flight of the individual fragments were then measured at angles from $0^{\circ}$ to $10^{\circ}$ relative to the direction of the primary beam. From this information, the fragmentation yields, differential in angle and energy, could be obtained for every element.

For this carbon experiment, the SHIELD-HIT simulations were split up into two parts. First, a simplified simulation to find the total fragmentation yields has been 
performed. Here, everything in the beamline, up to and including the water phantom, was simulated. But, instead of simulating the detector geometry explicitly a filter was added to the detection code, ensuring that only particles in the forward $10^{\circ}$ were scored in the phantom. To compensate for the lacking exit window, the simulation data was shifted $8 \mathrm{~mm}$ backwards when comparing with the experimental data. For the simulation of the angular and energy differential data, the full experimental geometry was simulated. The detector was modelled as cylinder shells, to exploit the experimental cylindrical symmetry.

The experiment has previously been used in benchmark studies of Monte Carlo transport codes (Böhlen et al. 2010, Pshenichnov et al. 2010). Here, FLUKA and Geant4 were compared with the experimental data. For Geant4, two different models of nuclear fragmentation were tested. First, the Binary Cascade light ion (BIC), which is a cascade model similar in concept to the Dubna Cascade Model used in SHIELD-HIT. The second is a quantum molecular dynamics model (QMD), which is similar to the model used by FLUKA. Like SHIELD-HIT, FLUKA and Geant4 use a de-excitation step with Fermi-breakup, though with different implementations.

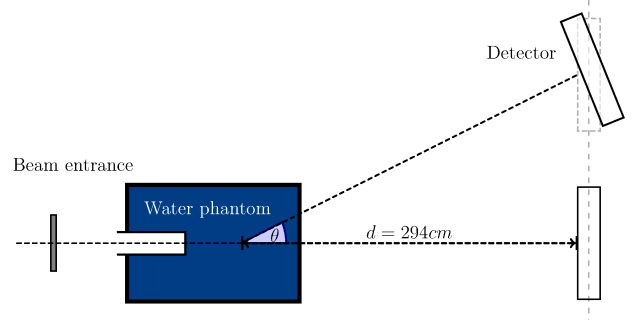

Figure 7: Setup of experiment

(Haettner 2006) used for validation of SHIELD-HIT10A. The ${ }^{12} \mathrm{C}$ ions hit a water target, and fragments are measured at an angle $\theta$ from the particle entry.

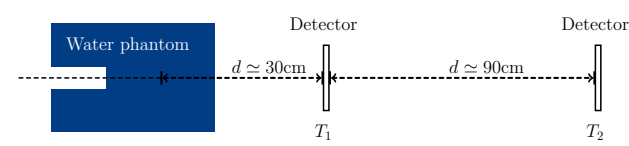

Figure 8: Setup of experiment

(Schimmerling et al. 1989) used for validation of SHIELD-HIT10A. The ${ }^{20} \mathrm{Ne}$ ions hit a water target, and fragments are measured on the beam-axis.

Earlier, a similar experiment was done at Berkley (Schimmerling et al. 1989). Here, a $670 \mathrm{MeV} / u{ }^{20} \mathrm{Ne}$-beam was shot at a variable size water phantom. The velocity and type of fragment were measured on the beam axis, using two time of flight detectors with radius $0.95 \mathrm{~cm}$. The exact placement of the objects in the beamline were not given in (Schimmerling et al. 1989). However, a distance of about $120 \mathrm{~cm}$ was reported in an earlier work of the same authors (Schimmerling et al. 1986) and assumed here in the simulation. For the neon experiment, only the water phantom itself was simulated, as the positions of the rest of the beamline elements were not described in (Schimmerling et al. 1989). The beam energy when hitting the phantom was $626.25 \mathrm{MeV} / u$ with an full width at half maximum (FWHM) of $5 \mathrm{~cm}$, as per (Schimmerling et al. 1989). Like the simulation of the carbon experiment, a filter was added to the detection code so 

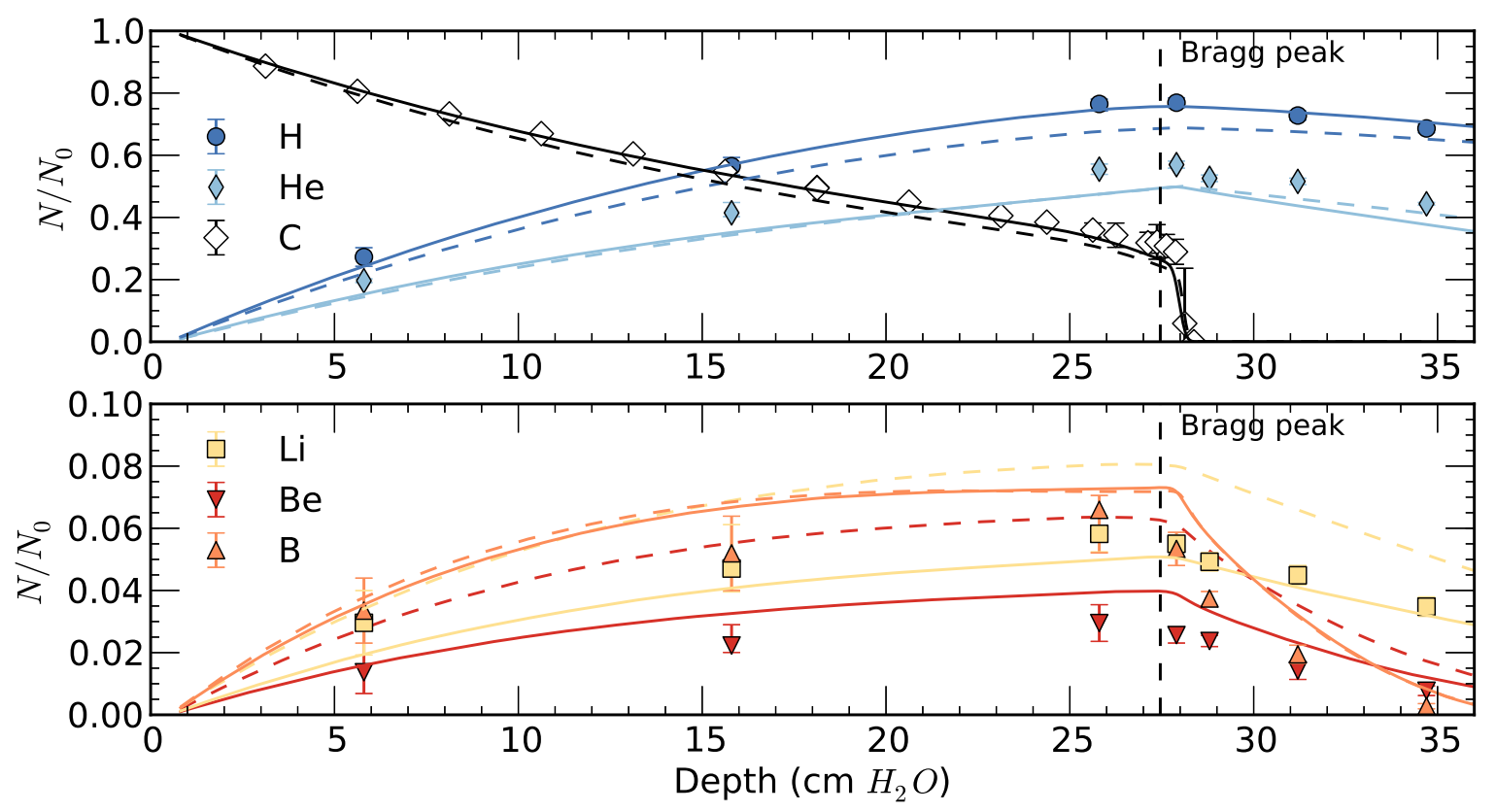

Figure 9: Relative yield of fragments emitted within a $10^{\circ}$ forward angle from a $400 \mathrm{MeV} / u^{12} \mathrm{C}$ beam in water, compared with experimental data (Haettner et al. 2006). Dashed line: SHIELD-HIT08simulation. Solid line:

SHIELD-HIT10Asimulation. The markers are experimental data. Where error bars are not visible, they are smaller than the markers.

only particles hitting both parts of the time of flight detector $\left(T_{1}\right.$ and $T_{2}$ on figure 8$)$ were scored.

For all simulations $4 \cdot 10^{7}$ primary particles were used.

\subsection{Comparison with fragmentation yields}

The total fragmentation yields from a $400 \mathrm{MeV} / u{ }^{12} \mathrm{C}$ beam in water are shown in figure 9. For the carbon ions SHIELD-HIT10A shows agreement within $2 \sigma$ of the experimental data, while SHIELD-HIT08 shows in general a lower fluence, consistent with the overestimated inelastic cross section seen in figure 4. The slight disagreement between SHIELD-HIT10A and the experimental data is probably due to boron fragments being misidentified as carbon in the experiment (Haettner et al. 2006). SHIELD-HIT10A shows excellent agreement with the hydrogen fragments before the Bragg peak, but a slight overestimation after. SHIELD-HIT08 generally underestimates the number of hydrogen fragments. Both codes underestimate the amount of helium produced, on the order of 15\%. For beryllium and lithium, SHIELD-HIT08 is greatly overestimating the number of produced fragments being consistent with the partial charge-changing cross sections in section 3.2.1. SHIELD-HIT10A is within $2 \sigma$ for the lithium fragments, but is overestimating the beryllium fragments somewhat, in particular after the Bragg peak. A similar scenario is seen for both SHIELD-HIT10A and SHIELD-HIT08 with 
the boron fragments, where the estimation is generally too high. For SHIELD-HIT10A this is particularly surprising, as the partial charge-changing cross sections for the boron channel fitted the experimental data very well. It has however been suggested that some underestimation is done by the experiment for this channel, as it was partly masked by the primary carbon ions (Haettner et al. 2006). This cannot be the sole source of the problem, however, as the problem continues beyond the Bragg peak, where all the primaries are gone. It could in part be caused by an overestimation of the boron channel below $100 \mathrm{MeV} / u$, where the partial-charge changing cross section predicted by SHIELD-HIT10A is much larger than at higher energies.

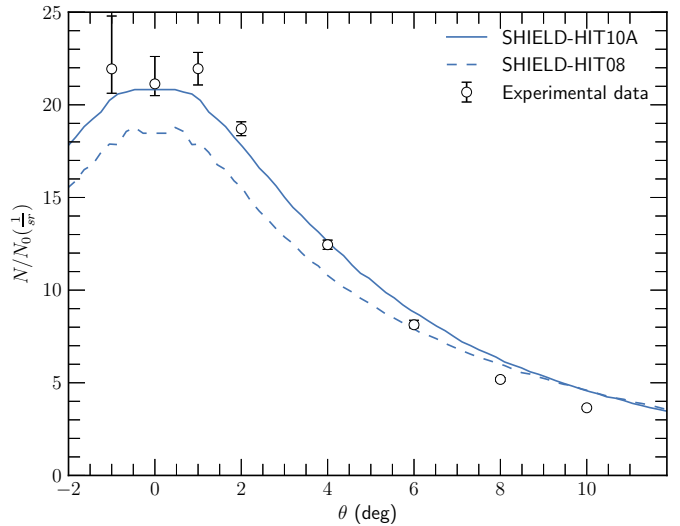

Figure 10: Angle resolved relative yield of hydrogen fragments at a depth of $25.8 \mathrm{~cm}$ water from a $400 \mathrm{MeV} / u^{12} \mathrm{C}$ beam, compared with experimental data

(Haettner 2006)

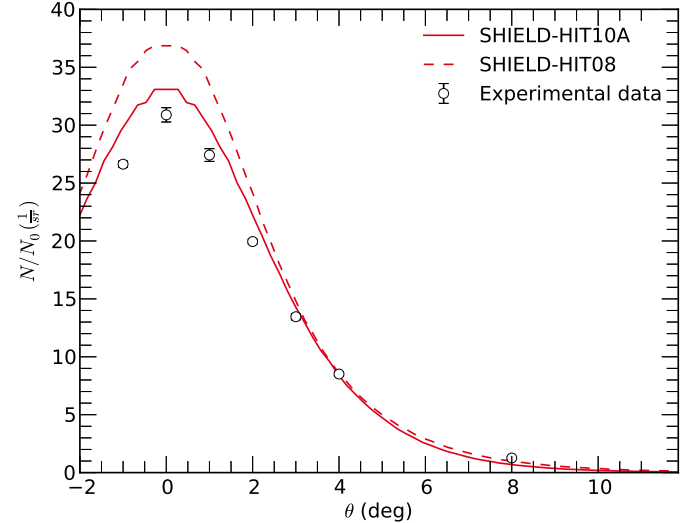

Figure 11: Angle resolved relative yield of helium fragments at a depth of $34.7 \mathrm{~cm}$ water from a $400 \mathrm{MeV} / u^{12} \mathrm{C}$ beam, compared with experimental data

(Haettner 2006)

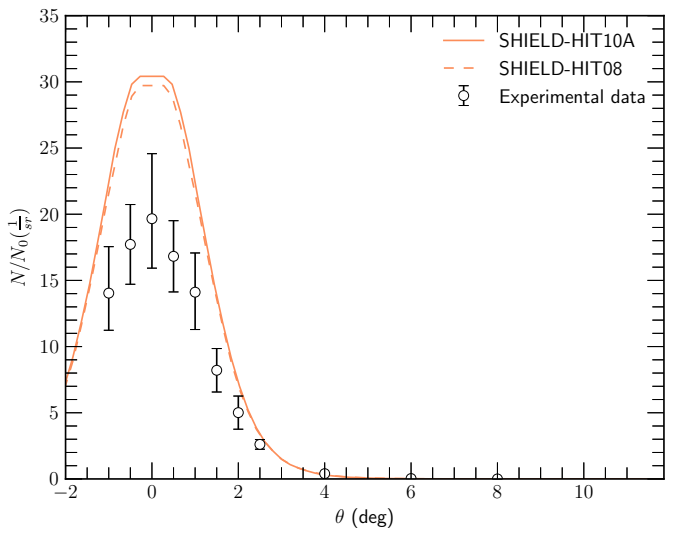

Figure 12: Angle resolved relative yield of boron fragments at a depth of $25.8 \mathrm{~cm}$ water from a $400 \mathrm{MeV} / u{ }^{12} \mathrm{C}$ beam, compared with experimental data (Haettner 2006)

Only some of the data compared with the experiment is explicitly discussed here. 

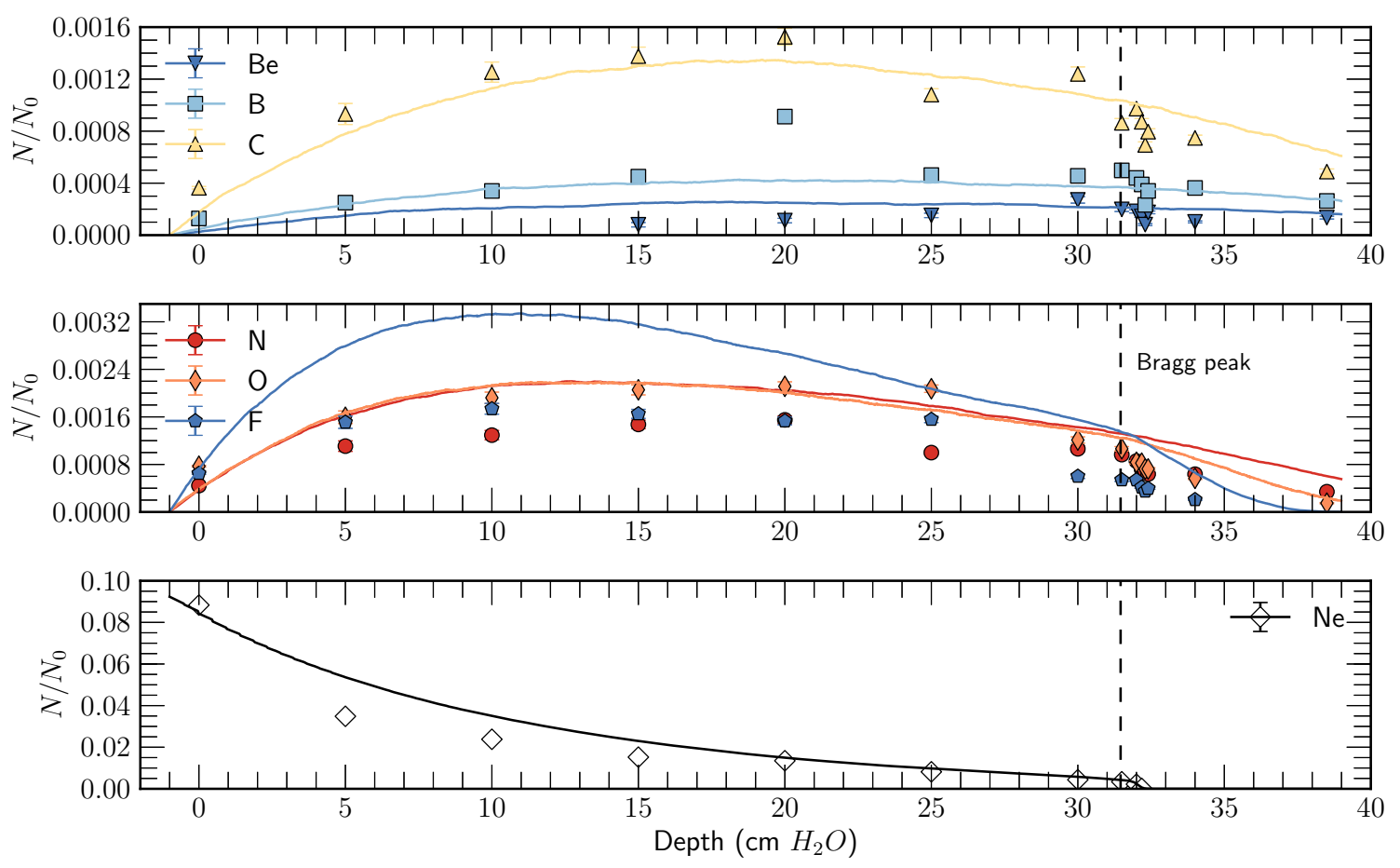

Figure 13: Relative yield of fragments from a $670 \mathrm{MeV} / u{ }^{20} \mathrm{Ne}$ beam in water compared with experimental data (Schimmerling et al. 1989). The solid line is the SHIELD-HIT10A simulation. The markers are experimental data. Where experimental error bars are not visible, they are smaller than the markers.

However, a comparison with all the data from the carbon experiment (Haettner 2006) is available in the online version of this article.

Looking at the angular resolved data (figures 10 and 11), SHIELD-HIT08 and to some extent SHIELD-HIT10A are underestimating the hydrogen fragments in the forward angles, while overestimating it at larger angles. The opposite effect is seen for helium, where both codes overestimate the small angles, but slightly underestimate the larger ones. Looking at the boron spectrum on figure 12, it is clear that the overestimation is mainly from the central angles, which is where the experimental boron yield might be masked by the primary carbon fragments.

For the fragmentation yield of neon, seen on figure 13, agreement with experimental data is in general not as good as that seen for carbon. As the neon experiment was simulated in less detail and detects only a smaller angular fraction of the fragments, this was to be expected. However, the discrepancies seen for neon in the lowest panel of figure 13 are surprising where the SHIELD-HIT10A simulation is consistently higher than the experiment. As neon is the primary particle, it would have relatively little angular divergence, and thus be less sensitive to setup errors. The difference might be caused by an overestimation of neon isotopes in SHIELD-HIT, as the other fragmentation yields would consistently be too low if the inelastic cross section was underestimated in general. 

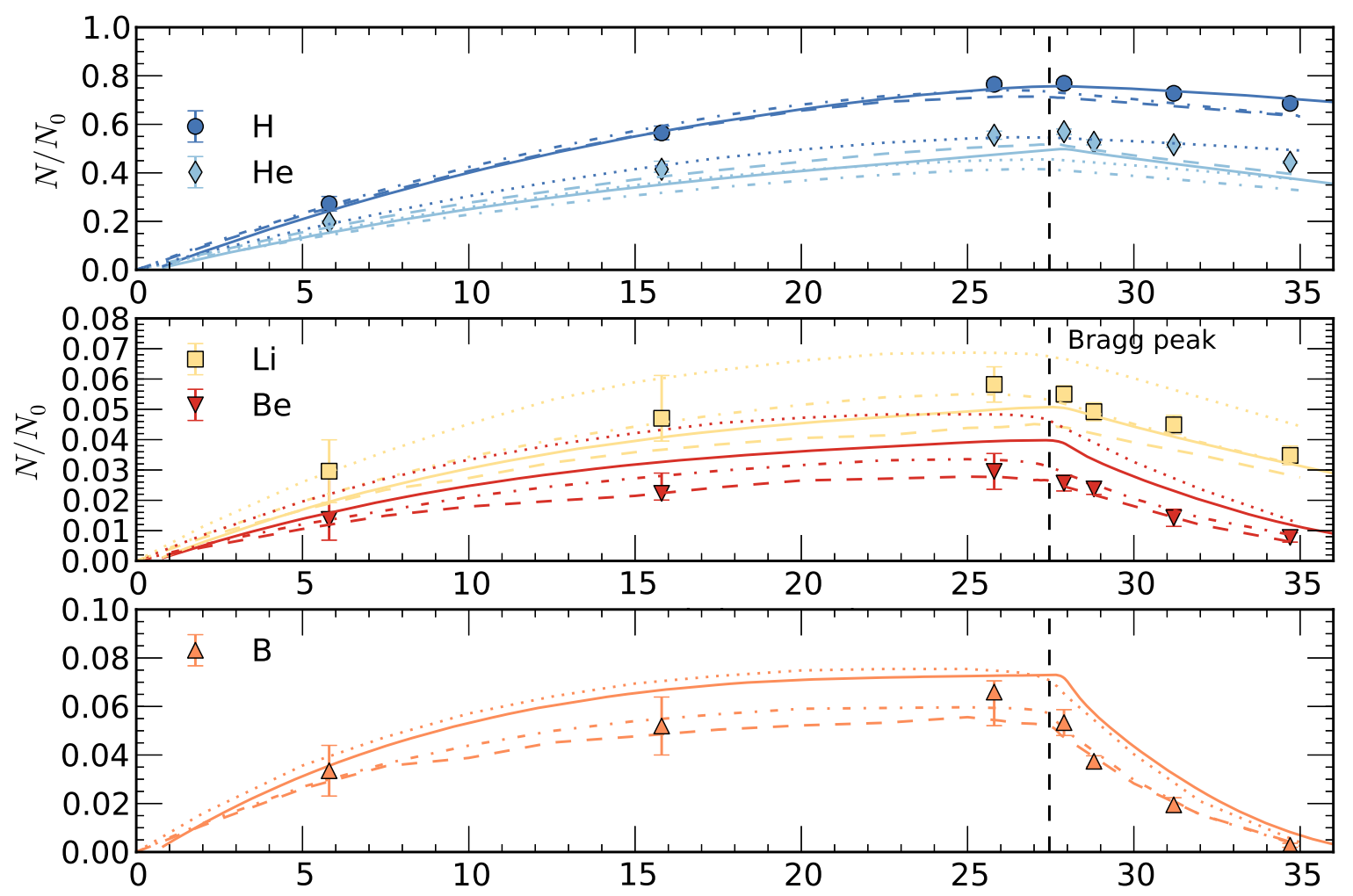

Figure 14: FLUKA, Geant4-QMD, Geant4-BIC LI (Böhlen et al. 2010) and SHIELD-HIT10A simulations of the relative yield of fragments emitted within a $10^{\circ}$ forward angle from a $400 \mathrm{MeV} / u{ }^{12} \mathrm{C}$ beam in water, compared with experimental data (Haettner et al. 2006). Dashed line: FLUKA simulation. Dashed-dotted line:

Geant4-QMD simulation. Dotted line: Geant4-BIC simulation. Solid line:

SHIELD-HIT10A simulation. The markers are experimental data. Where error bars are not visible, they are smaller than the markers.

For the lighter elements (Be, B and C), the agreement is generally good, though with some overestimation of the beryllium fragments far from the Bragg peak. For nitrogen, the yield is generally overestimated by SHIELD-HIT10A, though agreement is good for the oxygen yield. The largest overestimation is seen for the fluorine yield, which could be the result of an inaccurate angular distribution in SHIELD-HIT10A. It is interesting that for both neon and carbon ions, some discrepancies are seen for the $\Delta Z=1$ channel. It is likely that these fragments stem from grazing collisions for which the fast cascade model is dominating. While SHIELD-HIT10A presents in general the right order of magnitude for all fragments, further investigations should be made before SHIELD-HIT10A is used for simulations of neon beams which require very high accuracy.

\subsection{Comparison with other Monte Carlo Codes}

When comparing SHIELD-HIT10A with other Monte Carlo codes (figure 14), some tendencies can be observed. For hydrogen, helium and lithium, SHIELD-HIT10A 
shows similar agreement to FLUKA and Geant4-QMD, but without the tendency to underestimate the hydrogen fragments after the Bragg-peak. In addition, SHIELDHIT10A does not have the underestimation of helium seen with Geant4-QMD, nor the underestimation of lithium seen with FLUKA. For the heavier boron and beryllium fragments, however, FLUKA and Geant4-QMD show significantly better agreement with experimental data than SHIELD-HIT10A. For boron, it is particularly interesting to note how similar the Geant4-BIC model is to SHIELD-HIT10A. This suggest that the overestimation may be general to the cascade models, and it would be interesting to compare this with other codes using cascade models, such as MCNPX. None of the Monte Carlo codes show the best agreement with experimental data everywhere, though Geant4-BIC shows the largest discrepancies, as previously reported in (Böhlen et al. 2010). SHIELD-HIT10A shows similar or better agreement than FLUKA and Geant4-QMD for the light fragment yields, but also presents some discrepancies for the heavier fragments.

\section{Conclusion}

In this work, the improved nuclear models in SHIELD-HIT10A were presented and compared with a wide range of experimental data for nuclear interactions, as well as to the previous version of SHIELD-HIT (SHIELD-HIT08) and other Monte Carlo codes. SHIELD-HIT08 showed some overestimation for the total nuclear reaction cross section above $100 \mathrm{MeV} / u$, which was corrected in SHIELD-HIT10A. The new calibration of the models for nuclear fragmentation in SHIELD-HIT10A showed significantly improved agreement with experiments for the partial charge-changing cross sections for carbon on various materials, with an agreement that was in general within $2 \sigma$. When compared with fragmentation yield experiments of carbon ions on a water phantom, SHIELDHIT10A showed good agreement with the data. The agreement was comparable to that obtain in (Böhlen et al. 2010) with the Monte Carlo codes FLUKA and Geant4. While some discrepancies were observed for the SHIELD-HIT10A boron and beryllium yields the code showed similar or better agreement than FLUKA and Geant4 for the light fragments. When compared with fragmentation yield experiments of neon ions on water, larger discrepancies were observed, although SHIELD-HIT10A was in general in the right order of magnitude. In general, SHIELD-HIT10A is well suited for the simulation of problems arising in particle therapy, providing fast and accurate data. In order to further improve the nuclear models of SHIELD-HIT, more experimental data is required, in particular for energies below $100 \mathrm{MeV} / u$. Here the cross sections are the largest and the particles have the highest linear energy transfer, which is directly related to the biological effect of particle therapy. 


\section{Acknowledgments}

The authors wish to thank T.T.Böhlen for fruitful discussions on Monte Carlo simulations of fragmentation yield experiments and making data available in digital form. In addition we wish to thank M. Takechi for providing experimental data and M. H. Mortensen for digitizing data only available in print form.

\section{References}

Agostinelli S et al. 2003 Nucl. Instrum. Methods Phys. Res. A 506(3), 250-303.

Allison J et al. 2006 IEEE Transactions on Nuclear Science 53(1), 270-278.

Amelin N, Gudima K \& Toneev V 1990 Sov. J. Nucl. Phys 52, 172-178.

Barashenkov V 1993 JINR, Dubna pp. 1-346.

Bassler N, Hansen J W, Palmans H, Holzscheiter M H, Kovacevic S \& the AD-4/ACE Collaboration 2008 a Nucl. Instrum. Methods Phys. Res. B 266, 929-936.

Bassler N \& Holzscheiter M H 2009 Acta Oncol. 48, 223-226.

Bassler N, Holzscheiter M H, Jäkel O, Kovacevic S, Knudsen H V \& the AD-4/ACE Collaboration 2008 b Phys. Med. Biol. 53, 793-805.

Bassler N, Holzscheiter M H \& Petersen J B 2010 a Acta Oncol. 49, 1149-1159.

Bassler N, Kantemiris I, Engelke J, Holzscheiter M \& Petersen J B $2010 b$ Radiother. Oncol. 95, 87-93.

Battistoni G, Muraro S, Sala P, Cerutti F, Ferrari A, Roesler S, Fassò A \& Ranft J 2007 in M Albrow \& R Raja, eds, 'Proceedings of the Hadronic Shower Simulation Workshop 2006' Vol. 896 of AIP Conference Proceeding pp. 31-49. Fermilab 6-8 September 2006.

Berger M J, Coursey J S, Zucker M A \& Chang J 2005 'ESTAR, PSTAR, and ASTAR: Computer Programs for Calculating Stopping-Power and Range Tables for Electrons, Protons, and Helium Ions (version 1.2.3)'. National Institute of Standards and Technology, Gaithersburg, MD.

Bethe H A 1953 Phys. Rev. 89(6), 1256-1266.

Böhlen T T, Cerutti F, Dosanjh M, Ferrari A, Gudowska I, Mairani A \& Quesada J M 2010 Phys. Med. Biol. 55, 5833.

Bondorf J P, Botvina A S, Iljinov A S, Mishustin I N \& Sneppen K 1995 Physics Reports 257(3), 133 $-221$.

Botvina A, Dementyev A, Smirnova O, Sobolevsky N \& Toneev V 1997 International Codes and Model Intercomparison for Intermediate Energy Activation Yields, (by R. Michel, P. Nagel), NSC/DOC (97)-1, NEA/PET 14.

Botvina A S, Iljinov A S, Mishustin I N, Bondorf J P, Donangelo R \& Sneppen K 1987 Nuclear Physics A 475(4), $663-686$.

Dementyev A V \& Sobolevsky N M 1999 Radiat. Meas. 30(5), 553-557.

Fang D Q et al. 2000 Phys. Rev. C 61(6), 064311.

Fano U 1963 Annu. Rev. Nucl. Sci. 13, 1.

Fassò A, Ferrari A, Ranft J \& Sala P R 2005 'FLUKA: a multi-particle transport code' CERN-2005-10, INFN/TC_05/11, SLAC-R-773.

Fermi E 1950 Progress of Theoretical Physics 5(4), 570-583.

Geithner O, Andreo P, Sobolevky N, Hartmann G \& Jäkel O 2006 Phys. Med. Biol. 51, 2279-2292.

Golovchenko A, Skvarč J, Yasuda N, Giacomelli M, Tretyakova S, Ilić R, Bimbot R, Toulemonde M \& Murakami T 2002 Physical Review C 66(1), 14609.

Gudima K K, Mashnik S G \& Toneev V D 1983 Nuclear Physics A 401(2), 329 - 361.

Gudowska I, Sobolevsky N, Andreo P, Belkić D \& Brahme A 2004 Phys. Med. Biol. 49, 1933-1958.

Haettner E 2006 Experimental Study on Carbon Ion Fragmentation in Water Using GSI Theraphy Beams Royal Institute of Technology.

Haettner E, Iwase H \& Schardt D 2006 Radiation protection dosimetry 122(1-4), 485. 
Hall E J 2006 Int. J. Radiat. Oncol., Biol., Phys. 65, 1-7.

Hansen D C, Lühr A, Herrmann R, Sobolevsky N \& Bassler N 2012 Int. J. Rad. Biol. 88(1-2), 195-199.

Henkner K, Bassler N, Sobolevsky N \& Jäkel O 2009 Med. Phys. 36, 1230-5.

Hubert F, R. R \& Gauvin H 1989 Nucl. Instrum. Methods Phys. Res. B 36, 357.

IAEA TRS-398 2000 Absorbed dose determination in external beam radiotherapy an international code of practice for dosimetry based on standards of absorbed dose to water Technical report International Atomic Energy Agency.

ICRU Report 491993 Stopping powers and ranges for protons and alpha particles International Commission on Radiation Units and Measurements Bethesda, MD.

ICRU Report 732005 J. ICRU 5, 1-253.

Iwase H, Niita K \& Nakamura T 2002 JNST 39, 1142-51.

Kox S, Gamp A, Cherkaoui R, Cole A, Longequeue N, Menet J, Perrin C \& Viano J 1984 Nuclear Physics A 420(1), 162 - 172.

Kox S, Gamp A, Perrin C, Arvieux J, Bertholet R, Bruandet J, Buenerd M, Masri Y E, Longequeue N \& Merchez F 1985 Physics Letters B 159(1), 15 - 18.

Kox S et al. 1987 Phys. Rev. C 35, 1678.

Krämer M, Jäkel O, Haberer T, Kraft G, Schardt D \& Weber U 2000 Phys. Med. Biol. 45, 3299-3317.

Krämer M \& Scholz M 2000 Phys. Med. Biol. 45, 3319-3330.

Lindhard J \& Scharff M 1961 Phys. Rev. 124(1), 128-130.

Lühr A, Hansen D C, Jäkel O, Sobolevsky N \& Bassler N 2011a Phys. Med. Biol. 56, 2515-2533.

Lühr A, Hansen D C, Sobolevsky N, Palmans H, Rossomme S \& Bassler N 2011 b Acta Oncol. 50(6), 797805.

Lühr A, Toftegaard J, Kantemiris I, Hansen D C \& Bassler N 2012 Int. J. Rad. Biol. 88(1-2), 209-212.

Niita K, Sato T, Iwase H, Nose H, Nakashima H \& Sihver L 2006 Radiation Measurements 41(910), 1080-1090.

Parodi K et al. 2007 International Journal of Radiation Oncology*Biology*Physics 68(3), 920 - 934.

Paul H \& Schinner A 2001 Nucl. Instrum. Methods Phys. Res. B 179, 299.

Paul H \& Schinner A 2002 Nucl. Instrum. Methods Phys. Res. B 195, 166.

Pelowitz D B 2005 MCNPX users manual Los Alamos National Laboratory. [Online] Available: http://mcnpx.lanl.gov.

Powell M 1973 Mathematical Programming 4(1), 193-201.

Pshenichnov I, Botvina A, Mishustin I \& Greiner W 2010 Nucl. Instrum. Methods Phys. Res. B 268(6), 604-615.

Schaffner B, Pedroni E \& Lomax A 1999 Physics in Medicine and Biology 44(1), 27.

Schimmerling W, Miller J, Wong M, Rapkin M, Howard J, Spieler H \& Jarret B 1989 Radiation research $\mathbf{1 2 0}(1), 36-71$.

Schimmerling W, Rapkin M, Wong M \& Howard J 1986 Medical Physics 13(2), 217-228.

Schwaller P, Pepin B et al. 1979 Nuclear Physics A 316(3), 317-344.

Sihver L, H.Tsao C, Silberberg R, Kanai T \& Barghouty A F 1993 Phys. Rev. C 47, 1225.

Takechi M et al. 2009 Phys. Rev. C 79(6), 061601.

Toftegaard J, Lühr A \& Bassler N 2010 'libdEdx'. [Online] Available: http://libdedx.sf.net [27 February 2012].

Toneev V \& Gudima K 1983 Nuclear Physics A 400, 173 - 189.

Toshito T et al. 2007 Physical Review C 75(5), 54606.

Tripathi R, Cucinotta F \& Wilson J 1999 Nucl. Instrum. Methods Phys. Res. B 155(4), 349-356.

Tripathi R K, Cucinotta F A \& Wilson J W 1996 Nucl. Instrum. Methods Phys. Res. B 117, 347.

Tripathi R, Wilson J \& Cucinotta F 1997 Nucl. Instrum. Methods Phys. Res. B 129(1), 11-15.

Xu X G, Bednarz B \& Paganetti H 2008 Phys. Med. Biol. 53, R193-R241.

Zhang H Y et al. 2002 Nuclear Physics A 707(3-4), 303-324. 